\title{
Onset time of binocular rivalry and duration of inter-dominance periods as psychophysical markers of ADHD
}

\author{
J Antonio Aznar Casanova1,3, Juan Antonio Amador Campos ${ }^{2,3}$, Manuel Moreno \\ Sánchez ${ }^{1}$, Hans Supèr ${ }^{1,3,4} \S$ \\ ${ }^{1}$ Department of Basic Psychology, Faculty of Psychology, University of Barcelona, Passeig \\ Vall d'Hebron, 171, 08035 Barcelona, Spain; ${ }^{2}$ Department of Personality, Assessment and \\ Psychological Treatments; ${ }^{3}$ Institute for Brain, Cognition and Behaviour (IR3C); ${ }^{4}$ Catalan \\ Institute for Research and Advanced Studies (ICREA); e-mail: hans.super@icrea.cat \\ Received 16 December 2011, in revised form 14 November 2012
}

\begin{abstract}
Attention deficit hyperactivity disorder (ADHD) is one of the main neurobiological disorders in young children. Despite its prevalence, current diagnosis is debated. In this study we tested whether measures of binocular rivalry (BR) can contribute to the diagnosis of ADHD. BR is a phenomenon that is produced when two different images are presented to the two eyes simultaneously. Under these conditions the image presented to one eye competes with that presented to the other eye in seeking to achieve perceptual dominance. This competition is resolved through the activation of a given percept coupled with the suppression of the percept that had predominated until that point. We assume that the difficulty with inhibiting responses of ADHD children also affects their ability to inhibit the dominant image in a BR context. We analyzed the time to rivalry onset and the inter-dominance periods as measures of the temporal cost of resolving how long it takes for the brain to select (or suppress) one percept over the other. Our results show that the time to onset of rivalry (the first dominance) was longer in the clinical groups (ADHD-C and ADHD-I) than in the control group. As regards the interdominance periods, these were longer in the ADHD-C group than among controls, with the shortest period corresponding to the ADHD-I group. This study shows that BR can be used as a tool to develop a behavioral indicator of ADHD.
\end{abstract}

Keywords: attention deficit hyperactivity disorder (ADHD), binocular rivalry, inhibition mechanism, suppression/fusion mechanism, visual attention

\section{Introduction}

Attention deficit hyperactivity disorder (ADHD) is a neurobiological disorder that is estimated to occur in 3\%-7\% of school-age children (APA 2002). It is characterized by a low degree of attention, a high degree of hyperactivity and impulsivity, and the inability to inhibit inappropriate actions. The fourth revised edition of the Diagnostic and Statistical Manual of Mental Disorders (DSM-IV-TR) (APA 2002) distinguishes between three types of ADHD: predominantly inattentive (ADHD-I), predominantly hyperactive-impulsive (ADHD-HI), and combined (ADHD-C). Some researchers, however, believe that ADHD-I and ADHD-C are not variants of the same disorder, but rather different disorders, as they differ in the type of processes and altered brain circuits associated (Barkley 1997; Diamond 2005). One of the most influential models of ADHD proposes that deficits in inhibitory control are the core symptoms in this disorder (Barkley 1997). Deficient performance by children and adults with ADHD on motor and oculomotor tasks supports the inhibitory control model (for reviews, see Anderson et al 2007; Lijffijt et al 2005; Nigg 2001; Oosterlaan et al 1998; Rommelse et al 2008; Sergeants et al 2002). 
Binocular rivalry (BR) is a phenomenon that is produced when corresponding regions of the two eyes are stimulated with different images, and where the process of fusion cannot take place (Levelt 1965; Wheatstone 1838). Under these conditions the pattern presented to the left eye competes with that presented to the right eye in seeking to achieve perceptual dominance, and only one of these patterns will be selected (and subsequently perceived) at any given point, with awareness of the other being temporarily suppressed (Levelt 1965; Mueller and Blake 1989). Helmholtz (1962) was a pioneer in favor of the role of attention in BR, and later studies have found that attention, both exogenous and endogenous, plays an important role in relation to the onset of BR (Chong and Blake 2006; Chong et al 2005; Hancock and Andrews 2007; Meng and Tong 2004; Mitchell et al 2004; Ooi and He 1999) and durations of dominances (Lee et al 1999).

Traditionally, two parameters related with BR are the alternation rate (frequency of switches in visual awareness) and duration of the exclusive dominances corresponding to each rival image. However, the competition to achieve visual awareness must be quantified in terms of measures related with inhibition/activation of the required processes - namely, the onset of perceptual alternation and how much time elapses between successive, exclusive dominances. The 'time to rivalry onset' and the 'inter-dominance periods' should be understood as measures of the temporal cost of resolving the dilemma - that is, how long it takes for the brain to select (or suppress) one percept over the other. This dilemma is resolved through the activation of a given percept, which immediately becomes the dominant one, coupled with the suppression of the percept that had predominated until that point.

We assume that the reported difficulty with inhibiting responses of ADHD children also affects their ability to inhibit the dominant image in a BR context, such that compared with controls they would need longer to achieve visual awareness of the first dominant image; and, in turn, the period between two successive dominances would also be longer. Therefore, the aim here is to determine whether participants with ADHD present higher values on both these measures. Given the nature of the task, any differences in performance would be attributable solely to endogenous factors - in other words, the BR measures would reveal characteristics of the participants.

The experiment conducted comprised two studies. Study 1 compared the latencies of the rivalry period (onset time of the first exclusive dominance) in four groups of subjects: ADHD-C, ADHD-I, and controls ( 1 and 2). This measure can be regarded as an indicator of the temporal cost of resolving the conflict - that is, how long it takes for the mechanism to select (activate) one of the two competing precepts and suppress (inhibit) the other. Study 2 then measured the time elapsed (duration) between two successive, exclusive dominances. This temporal measure must also be related with the ongoing struggle for selection/suppression (activation/inhibition) that is established between simultaneous stimulations of the two eyes, or between internal representations.

The data reveal that the onset time of the first exclusive dominance was longer in the ADHD-C and ADHD-I groups compared with the control group. The inter-dominance periods were longer in the ADHD-C group than among controls, with the shortest period corresponding to the ADHD-I group. In conclusion, this study shows that BR can be used as a tool to develop a behavioral indicator of ADHD.

\section{Method}

\subsection{Participants}

Seventy-two participants, aged between 6 and 14 years ( mean $=10.33$ years; SD $=2.71$ years), were assigned to three groups (ADHD-C, ADHD-I, and Control-1) according to the criteria described below. 
The ADHD-C group comprised twenty participants (fifteen males), while the ADHD-I group had twelve (ten males). They were all recruited through a public hospital in Barcelona. A clinician made the diagnosis according to DSM-IV-TR (APA 2002) criteria, through an interview (Clinical Interview-Parent Report form) (Barkley and Murphy 2006) and according to the results of the ADHD questionnaire (Amador Campos et al 2006) and the Conners's (1997) revised scales, which were answered by parents and teachers. The classification of ADHD subtype was based on both the results of the interview and questionnaire scores.

Participants were classified as ADHD-I if they fulfilled the criteria for inattention but not those for hyperactivity-impulsivity on both the interview and the questionnaires for parents and teachers (six or more symptoms of inattention and fewer than six symptoms of hyperactivityimpulsivity, scored as 2 or 3 on the ADHD questionnaire for parents and teachers; $t$-scores $>65$ on the cognitive difficulties/inattention factor and the ADHD index and $t<65$ on the hyperactivity factor of the Conners's scale for parents and teachers).

Participants with combined type (ADHD-C) fulfilled the criteria for both inattention and hyperactivity-impulsivity (six or more symptoms of inattention and hyperactivity-impulsivity, scored as 2 or 3 on the ADHD questionnaire for parents and teachers; $t$-scores $>65$ on the cognitive difficulties/inattention and hyperactivity factors and the ADHD index of the Conners's scale for parents and teachers).

The Control-1 group consisted of forty participants (seventeen males), all recruited through a private school in Barcelona. They all had fewer than six symptoms of inattention and hyperactivity-impulsivity, as assessed by parents and teachers using the ADHD questionnaire, and $t$-scores of less than 60 on the cognitive problems/inattention and hyperactivity factors and the ADHD index of the Conners's scales for parents and teachers. An additional adult's group (Control-2) composed of twelve undergraduate students, aged from 20 to 24 years, was added.

All participants had normal or corrected-to-normal visual acuity, as well as high-grade stereoacuity (higher than 60 seconds of arc), as assessed by the Titmus test. In all groups most $(>95 \%)$ of the participants were eye-right dominant.

The exclusion criteria for groups were: total IQ $<85$ according to the Wechsler Intelligence Scale for Children (4th edition) (WISC-IV) (Wechsler 2003/2005), a history of tics, Tourette's syndrome, neurological disorders (eg seizures, brain lesions, meningitis, or encephalitis), and several other disorders of relevance (autism, difficulties with learning, motor skills or communication, psychosis, bipolar disorder, and mood or anxiety disorders). Participants with ADHD who were taking stimulant medication $(n=7)$ were not excluded from the study, but they were assessed after a period of 24 hours without medication.

The study was approved by both the hospital's ethics committee and the management board of the school. All the participants volunteered to take part in the study and signed informed consent (adult's Control-2 group) or their parents or legal guardians (for children). The study was conducted in accordance with the Declaration of Helsinki of 1975 (as revised in Tokyo in 2004).

\subsection{Stimulus}

The stimulus used in the binocular rivalry task (BRT) was a bistable image (anaglyph) comprising two superimposed, coloured sine-wave (4 cycles of spatial frequency) gratings (see figure 1). The size of the image, as seen from a distance of $60 \mathrm{~cm}$, subtended a visual angle of $5 \mathrm{deg}$ on the observer's retina. So, the spatial frequency of the stimulus was 0.8 cycle $\mathrm{deg}^{-1}$. As regards the two overlapping layers in the bistable image, one contained a sinusoidal variation of luminance in the red spectral band (figure 1, bottom left), while the other had a variation of luminance in the green band (figure 1, bottom right). In half of the trials (randomly interleaved) the red-green grating was vertical-horizontal, and vice versa 
in the other half. The two overlapping images formed an anaglyph, which when presented dichoptically produced the phenomenon of BR. The transmittance of red and green filters were adapted to the red-green sine-wave gratings which had a wavelength of 700 and $550 \mathrm{~nm}$, respectively.

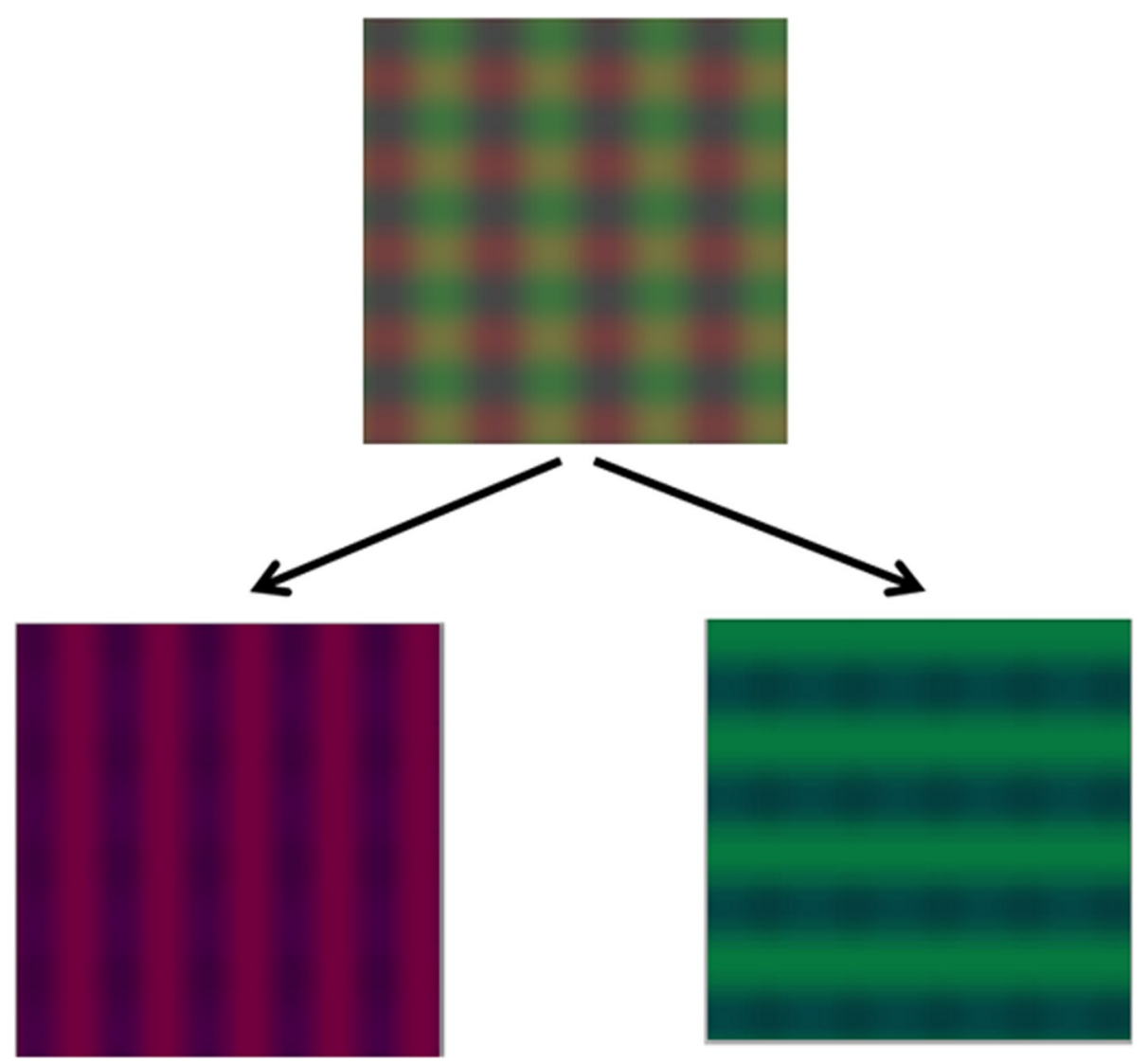

Figure 1. [In colour online, see http://dx.doi.org/10.1068/p7203] The bi-stable image used as a stimulus in the binocular rivalry task. The image comprises two layers (top panel). Separation of the two layers that make up the composite image: a sine-wave grating with variation of luminance in the red band (bottom left) and another grating with variation of luminance in the green band (bottom right).

\subsection{Apparatus}

The BRT was applied using a Lenovo ThinkPad T510 PC. The stimuli were presented on a 22 -inch screen, with a spatial resolution of $1024 \times 768$ pixels. Large eye movements and blinking were controlled by an eyetracker (iViewRED-120 Hz, SensoMotoric Instruments GMBH, Teltow, Germany). Red-green filter glasses were used to present the images dichoptically. The responses of participants were recorded by means of a response box, while a computer program (Experiment $\mathrm{Center}^{\mathrm{TM}}$, v.2.5 ${ }^{\circ}$, by SMI) controlled the time of stimuli presentation and the fixation point, and also recorded the number of alternations in dominance/suppression and the duration of exclusive dominance periods.

\subsection{Procedure}

The participants were all assessed in a room (office of the hospital, school, or college) that provided similar environmental conditions. They were sat $60 \mathrm{~cm}$ from the screen, at the centre of which were presented the anaglyph images designed for the BRT. They were instructed to look continuously at the centre of the image for $30 \mathrm{~s}$ (each trial), without moving their head or eyes, or blinking. These factors were controlled through the use 
of a chin-rest and an eyetracker. Each participant put on the red-green glasses, and the examiner then verified the participant's stereoscopic visual ability by presenting him or her with a different anaglyph. After the required calibration with the eyetracker, the test based on the BRT started (C) Aznar-Casanova 2010).

Each trial of the BRT began with the presentation of a white image, with a cross drawn at the centre, at which the observer had to look for $2 \mathrm{~s}$. The fixation cross was removed, and a stimulus/anaglyph, containing the two overlapping rival images (see figure 1), was then immediately presented and the participant had to continue staring at its centre (rival images) within a virtual window of $2 \mathrm{deg} \times 2 \mathrm{deg}$. The relatively large fixation window allowed the subject to make small eye movements, thereby preventing perceptual fading (Martinez-Conde et al 2009).

While the anaglyph was being displayed, participants had to press the response box button marked with vertical lines at the precise moment that he or she was aware of seeing only a vertical grating, and had to keep it pressed while watching. Similarly, they had to press the button marked with horizontal lines at the precise time that he or she became aware of seeing only a horizontal grid, and had to keep it pressed while watching. When none of the gratings (horizontal or vertical) clearly predominated, the subject was instructed not to press any button. Figure 2 shows the sequence of a trial in this task. Prior to applying the test, participants were trained in the modus operandi of the two response options by showing them as stimuli a horizontal and a vertical arrow.

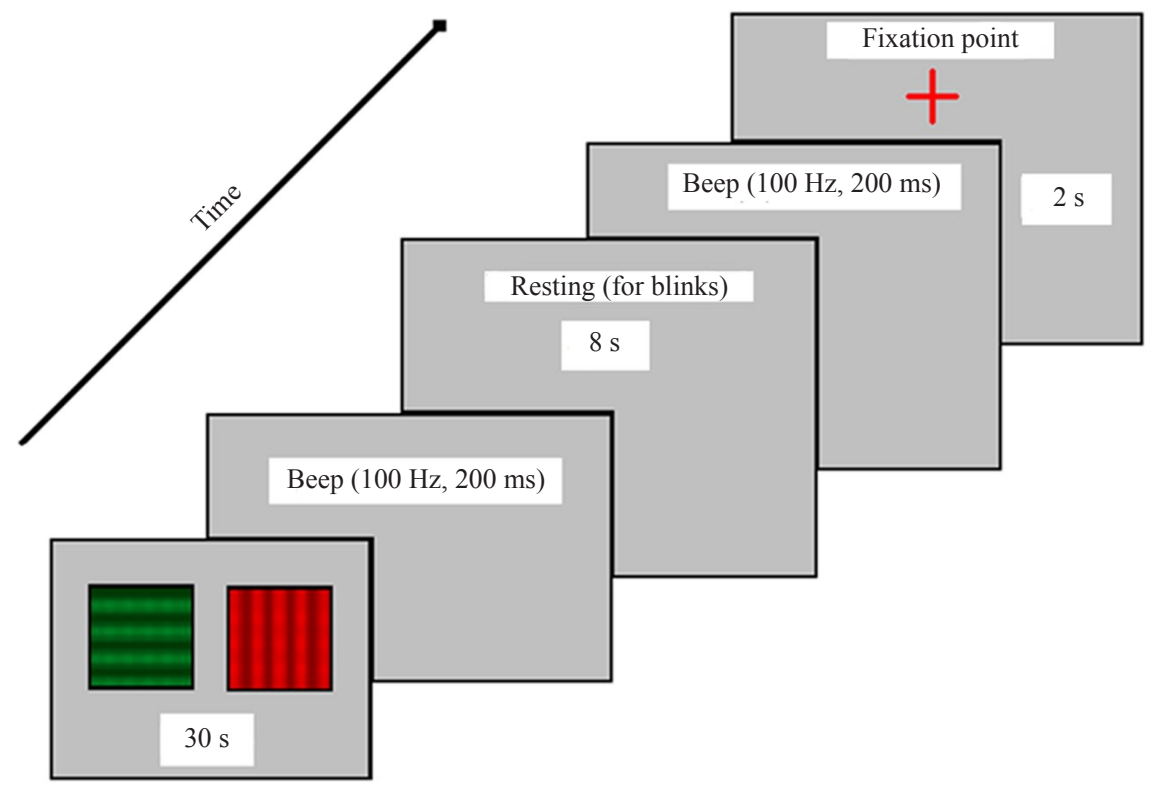

Figure 2. [In colour online.] Temporal sequence of a trial in the binocular rivalry task. The diagram shows both the fixation cross and the dichotic stimuli presented during the test. Note the small rest periods in which blinks are allowed.

For each trial we recorded the onset time of the rivalry period for each participant - that is, the time elapsed from the initial presentation of the stimulus-anaglyph until the observer pressed one of the two response buttons, indicating that either the horizontal or the vertical grating was being seen (see figure 3). However, we also recorded the duration of the time period between two successive dominances - that is, how long elapsed between suppression of horizontal grating perception until clear perceptual dominance of the vertical grating, or vice versa. Time spent blinking was excluded from the analysis, and only trials with calibrations $\leqslant 1^{\circ}$ were included. 


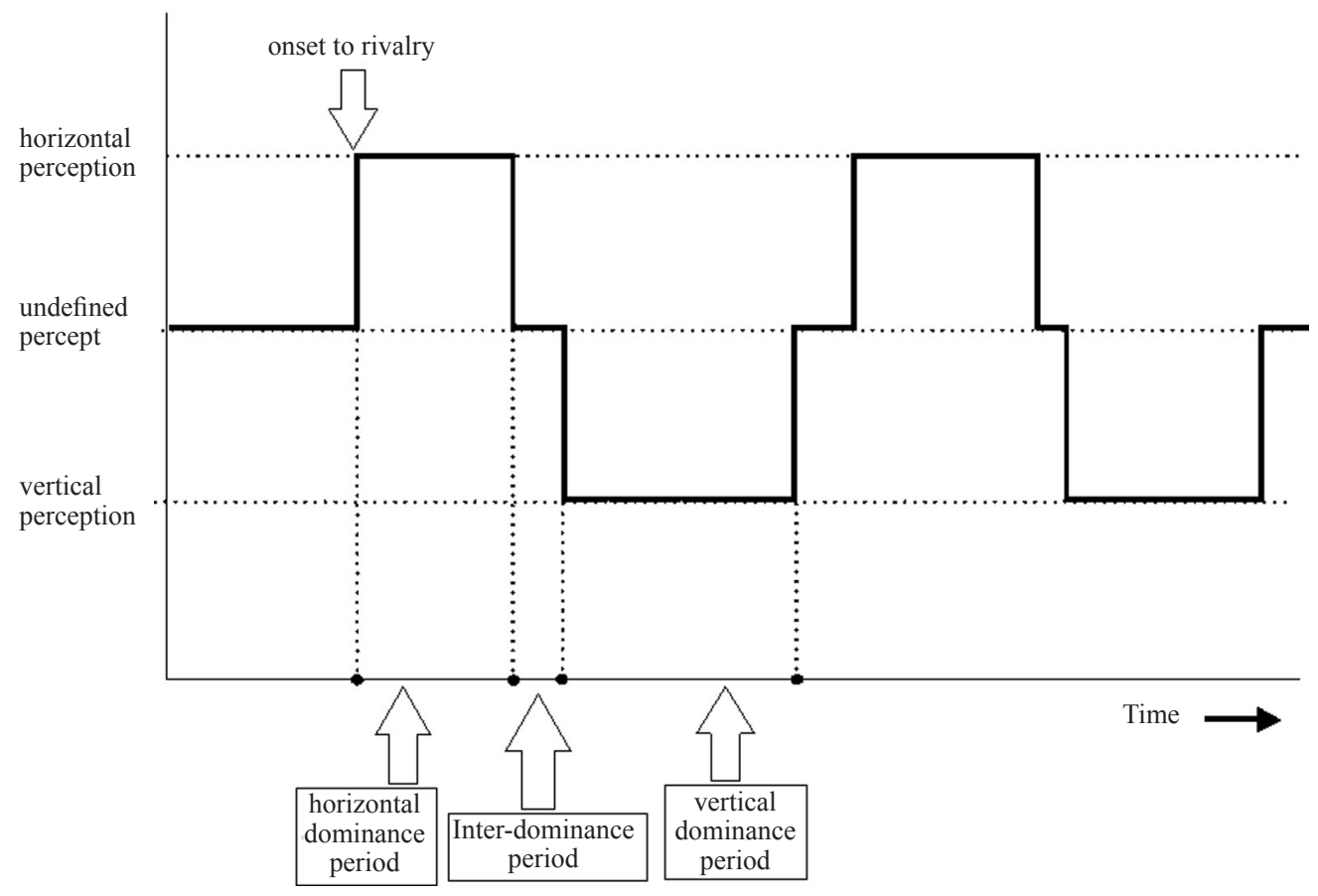

Figure 3. Geometric definition of the dependent measures in the binocular rivalry task: (1) the time to rivalry onset; and (2) the inter-dominance periods.

Each participant performed 10 trials of the BRT, each of which lasted $30 \mathrm{~s}$. Between two successive trials a white screen was displayed for $8 \mathrm{~s}$, followed by another screen which showed the fixation point for $2 \mathrm{~s}$. The total duration of the test, including training, was approximately $10 \mathrm{~min}$.

\section{Results}

3.1 Analysis of the onset time of the first exclusive dominance across trials

We first analyzed the onset latencies to the first dominance. Therefore, the means of the time to rivalry onset were calculated and entered into an ANOVA for 4 groups (ADHD-C, ADHD-I, Control-1, Control-2) with the variable 'age' as a covariate factor. Whenever a main effect reached significance, comparisons were made using Tukey tests.

The statistical analysis showed a significant effect of 'groups' $\left(F_{3,835}=29.30, p<0.001\right)$. The covariate factor 'age' was significant $\left(F_{1,835}=83.16, p<0.001\right)$. A posteriori comparisons (HSD Tukey test) indicated that the two clinical groups ADHD-C ( $M=2456.92 \mathrm{~ms}$, $\mathrm{SE}=138.6)$ and ADHD-I $(M=2041.68 \mathrm{~ms} ; \mathrm{SE}=179.3)$ achieved a statistically equivalent time of onset to the first dominance, but that this differed significantly from that of the Control-1 $(M=1706.6 \mathrm{~ms}, \mathrm{SE}=97.5)$ and Control-2 $(M=1527.7 \mathrm{~ms}, \mathrm{SE}=95.4)$ groups (see figure 4). Onset latencies to the first dominance were not significantly different between both control groups.

\subsection{Analysis of the time between successive, exclusive dominances across trials}

Next we examined the inter-dominance periods. For each trial, means of the inter-dominance periods on the BRT were calculated by dividing the duration of such inter-dominance periods by the alternation frequency. Then these periods from all trials were entered into an ANOVA for 4 groups (ADHD-C, ADHD-I, Control-1, Control-2), controlling for 'age' as a covariate factor. The ANOVA showed that the main effects of 'group' $\left(F_{3,835}=26.957, p<0.001\right)$ was significant. The covariate factor 'age' was also significant $\left(F_{1,835}=79.902, p<0.001\right)$. 


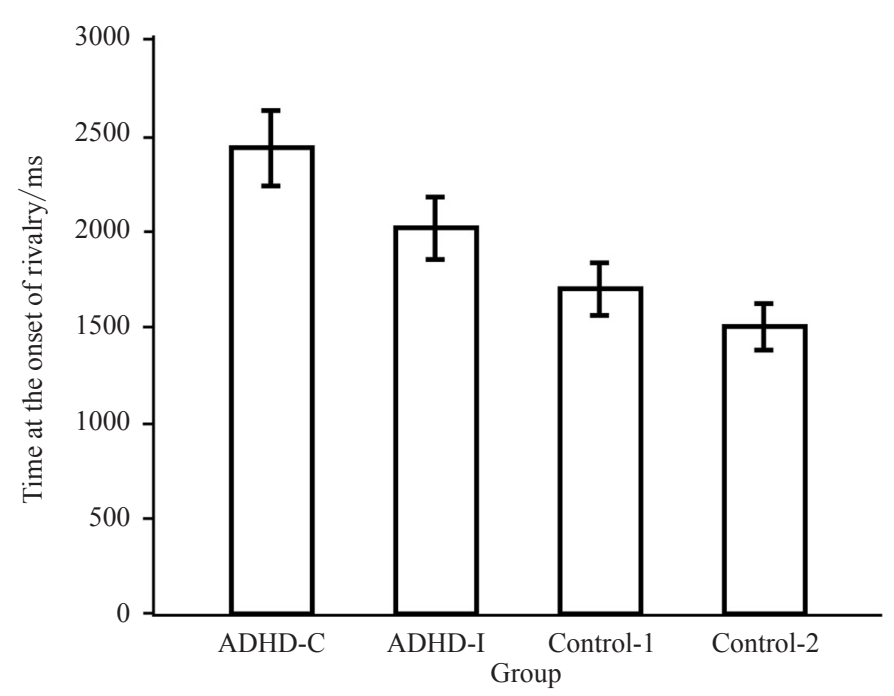

Figure 4. Comparison between the four groups (ADHD-C, ADHD-I, and Control-1 with equal age and Control-2 adults) as regards the time to rivalry onset. The bars show the $95 \%$ confidence interval.

A posteriori comparisons (Tukey test) revealed that the Control-1 group ( $M=536.68 \mathrm{~ms}$, $\mathrm{SE}=49.74)$, Control-2 group $(M=486.68 \mathrm{~ms}, \mathrm{SE}=63.71)$ and the ADHD-I groups $(M=459.64 \mathrm{~ms}, \mathrm{SE}=91.43)$ had similar inter-dominance periods, which differed significantly from that of the ADHD-C group $(M=742.45 \mathrm{~ms}, \mathrm{SE}=70.67)$ (see figure 5).

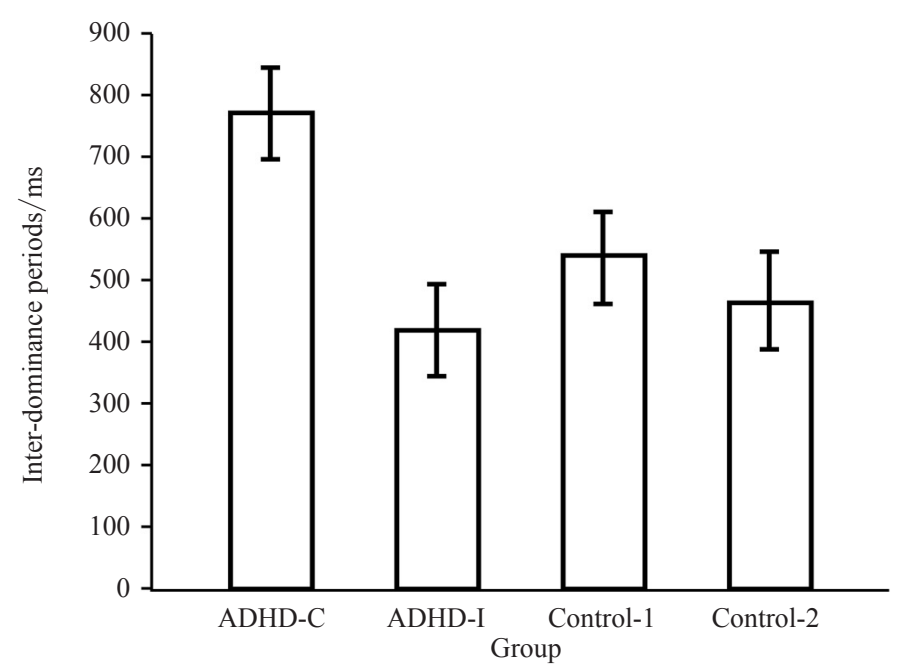

Figure 5. Comparison between the four groups (ADHD-C, ADHD-I, and Control-1 with equal age and Control-2 adults) as regards the inter-dominance periods. The bars show the $95 \%$ confidence interval.

\subsection{Analysis of processing speed}

Furthermore, we tested whether the three groups of children (ADHD-C, ADHD-I, and Control-1) were equivalent in processing speed. To this, subjects were selected according to their scores on the index of processing speed (PS) of the WISC-IV (Wechsler 2003/2005). This index combines scores of two tests, "Digit symbol-coding" and "Symbols search", both using visual stimuli. The average results are: ADHD-C $(M=102.7, \mathrm{SD}=11.14)$; ADHD-I $(M=101.9, \mathrm{SD}=14.13)$; Control-1 $(M=109.88, \mathrm{SD}=13.1)$. A posteriori comparisons (HSD Tukey) indicated that the two clinical groups and the Control-1 group achieved statistically equivalent scores. 


\subsection{Analysis of the alternation rate and exclusive dominance duration}

Finally, we calculated the alternation rate and dominance duration. For the measure (dependent variable) 'alternations' the variable 'groups' was significant $\left(F_{3,79}=7.693\right.$, $p<0.001)$. However, for the measure 'dominance duration' the variable 'groups' was not significant $\left(F_{3,79}=0.373, p<0.773\right)$. These results are in agreement with those obtained by Meng and Tong (2004). Figure 6 shows the mean duration of dominance periods separately for horizontal and vertical gratings. The differences in durations corresponding to the two percepts (horizontal and vertical gratings) were not statistically significant - that is, they spend a similar time at each grating.

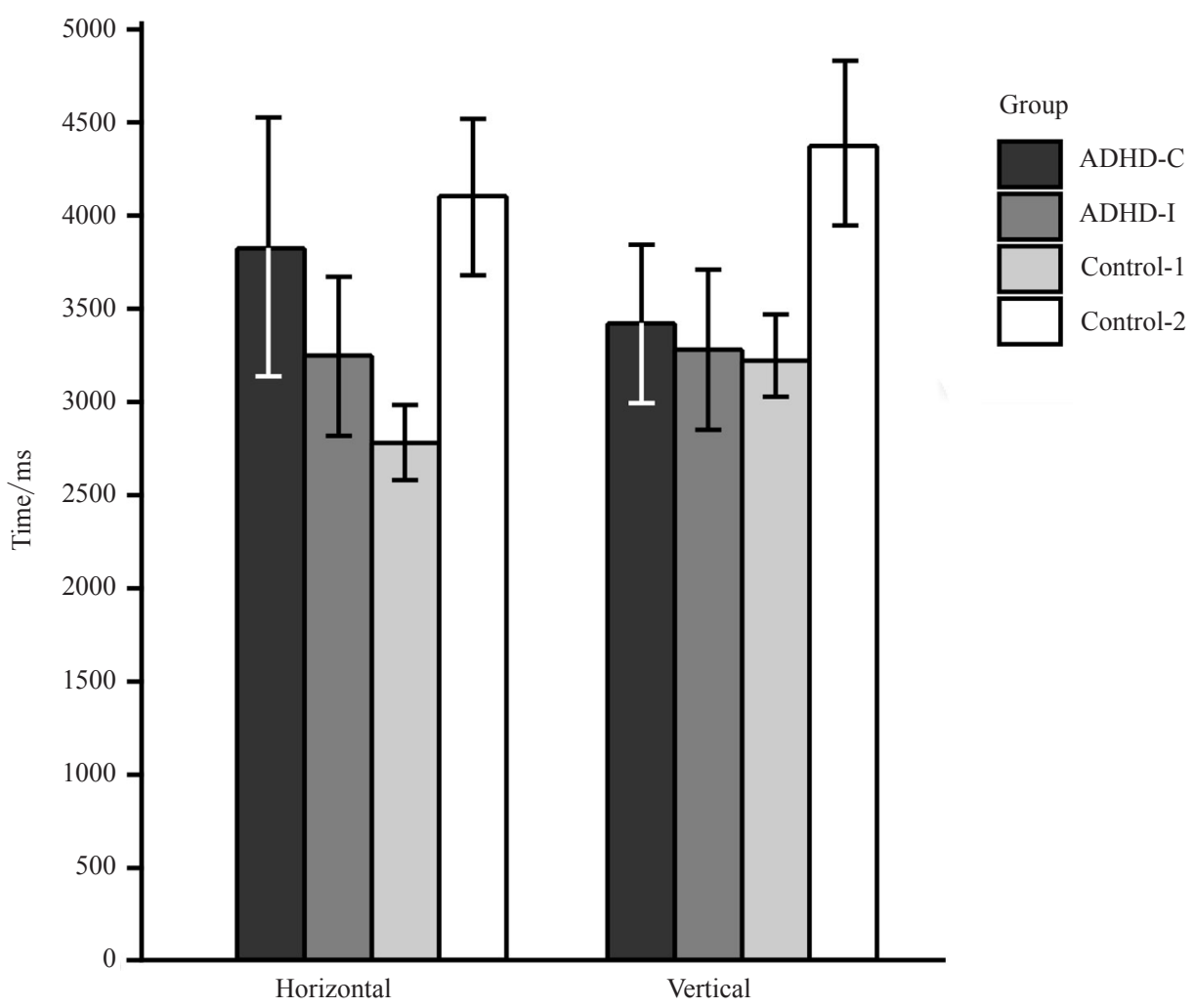

Figure 6. The means of the dominance duration for the horizontal and vertical gratings. The bars show the $95 \%$ confidence.

\section{Discussion}

This study used a BRT as an experimental paradigm to determine how long the brain takes to inhibit a currently dominant image and to activate a previously suppressed one. It was hypothesized that children with ADHD would find it more difficult than controls to resolve the conflict between activation and deactivation of a succession of percepts. A further assumption was that the time to the onset of alternating perceptual rivalry and the period between two successive dominances are related to how easy or difficult it is for the cognitive system to resolve this dilemma. Therefore, the time to rivalry onset and the duration of interdominance periods were used as measures of the interaction between the mechanism which activates the percept that is about to become dominant and the mechanism that inhibits the percept which is to be suppressed.

The analysis of data corresponding to the four study groups (ADHD-C, ADHD-I, and Control-1 and Control-2) showed, firstly, that the time to rivalry onset was greater in both clinical groups as compared with the control groups. Our observed onset times are longer than previous observations (Wolfe 1983). This may be explained by the different task settings. 
For instance, in the experiment of Wolfe afterimages were used to produce rivalry, and they included flashing stimuli, whereas our study consists of static images. Our results also show that the duration of transitional periods between successive dominances was greater in the ADHD-C group as compared with the remainder groups (ADHD-I, Control-1, and Control-2), whose mean values were equivalent. The results may be explained by slowed motor processes, as there are two components (sensory and motor) in the reaction time of the subjects, or by differences in decision making, which may vary between groups and subjects. However, the three groups of children were equivalents in the processing speed index of the WISC-IV, which measures the ability to process and discriminate visual information quickly and efficiently. Although we cannot be certain whether or not subjects in the ADHD-C group violate the criterion of perceptual dominance in the BRT, these results disagree with an explanation of slower motor processes. In line with this conclusion are neurophysiological findings that demonstrate that the variability in reaction times is explained by the variability in the integration of perceptual information, which is modulated by attention.

Several studies have found that attention, both exogenous and endogenous, plays an important role in relation to the onset of BR (Chong and Blake 2006; Chong et al 2005; Hancock and Andrews 2007; Meng and Tong 2004; Mitchell et al 2004; Ooi and He 1999). Furthermore, the manipulation of stimuli (exogenous attention) favours perceptual alternation and increases the duration of dominance periods (Hancock and Andrews 2007; Meng and Tong 2004; Mitchell et al 2004). In the present study the two rival stimuli were made equivalent in order to neutralize the influence of exogenous attention. As such, the longer time to rivalry onset observed among participants with ADHD can be due only to difficulties with endogenous attention.

During the last decades there has been a debate on whether BR emerges from competition between monocular neurons in V1 and other low-level associated areas (Blake 1989; Lehky 1988; Pettigrew 2001; Tong 2001) or between pattern-selective binocular neurons (representations) in higher level visual areas (Leopold and Logothetis 1996; Logothetis et al 1996; Sheinberg and Logothetis 1997). The finding of longer duration inter-dominance periods in the ADHD-C group as compared with the remainder groups suggests that BR is not completely resolved in low-level regions of the visual pathway (V1, V3, V4, V5) and that the mechanisms responsible for the activation and suppression of perception in the BRT must be allocated in higher level areas in the visual pathway. These results are also compatible with that of Lamme and Roelfsema (2000), who proposed an interactive model according to which V1 forms dynamic recurrent connections with high-level extra-striate areas, which are required for maintaining representations in awareness.

Some authors have reported the onset of rivalry to be independent of the sustained rivalry that is produced with long presentations of stimuli (Carter and Cavanagh 2007; Kalisvaart et al 2011). However, these studies explored ocular predominance at the onset of rivalry and the effects of stimulus contrasts or movement on the onset time and the maintenance of rivalry. The present study found that the time to rivalry onset was greater in both clinical groups (ADHD-C and ADHD-I) than in the control groups. The fact that individuals in the two ADHD groups took longer to reach the onset of rivalry could be due to less flexibility between the activator (excitatory) mechanism and the inhibitory mechanism when it comes to resolving the perceptual conflict.

The analysis of the duration of inter-dominance periods showed that the behaviour of the ADHD-I group was more similar to that of the control groups (Control-1 and Control-2) than that of the ADHD-C group. Although the two clinical groups share problems of attention, research has shown that there are differences between individuals classified as ADHD-C or ADHD-I: children with ADHD-I have greater difficulties with focused and selective attention, 
whereas those with ADHD-C find it more difficult to maintain attention and concentration (for a review see Barkley 2006). Some authors (Blake and Logothetis 2002; Meng and Tong 2004) have suggested that perceptual alternation may involve an attentional switch, such that in the BRT the selection of one percept among two possibilities requires a reflex (or involuntary) inhibition of one of them. Thus, the fact that individuals with ADHD-C need more time to inhibit a percept may indicate that they also have more difficulties with involuntary inhibition. Difficulties with the voluntary inhibition of behaviour are well documented among children and adults with ADHD-C (see Barkley 1997), but research has yet to study tasks that require reflex inhibition - hence the importance of the present data, which highlight the difference between the two clinical groups as regards the mechanism of automatic inhibition. It should be noted that the results cannot be attributed to the possibility that participants in the ADHD-I group were subclinical ADHD-C individuals, since ten of the twelve participants had three or fewer symptoms of hyperactivity-impulsivity, while the remaining two individuals had four (Milich et al 2001).

A final interesting finding is that each of these measures ('the onset time' and 'interdominance periods') provides information about complementary (rather than identical) aspects of attentional mechanisms. Specifically, the time to rivalry onset appears to refer to the 'attentional set' at the start of the BRT, whereas the inter-dominance periods seem to provide information about the amount of attentional resources needed and/or the strength of inhibition as the task proceeds and sustained attention is required. In conclusion, this pattern of results suggests that the clinical sample can be characterized, and distinguished from, controls, on the basis of the onset time and inter-dominance periods, both parameters being BR-related. These parameters have to do with the inhibitory mechanism underlying perceptual alternation in visual awareness. Further research is now required to investigate how other factors (related to the stimulus, the task, or the subject) may affect the two measures studied here.

The diagnosis of ADHD is based on clinical criterion and driven by interviews, symptom rating scales, psychometric, laboratory, and neuropsychological tests. However, the diagnosis of attention disorders is controversial, and currently the assessment of attention deficit has been questioned (Adams et al 2008; Carlson and Mann 2002; Diamond 2005; Milich et al 2001). Our results show that BR can be used as a behavioral indicator of attention deficit and therefore can contribute to the diagnosis of ADHD.

Acknowledgments. This work was funded by grants awarded by the Alicia Koplowitz Foundation (reference FAKO-2009) to J A Amador Campos, from the Spanish Ministry of Science and Innovation, to J A Aznar Casanova (reference PSI2009-11062/PSIC) and to H Supèr (reference PSI2010-18139), and from the Catalan government (reference 2009-SGR-308).

\section{References}

Adams Z W, Derefinko K J, Milich R, Fillmore M T, 2008 "Inhibitory functioning across ADHD subtypes: Recent findings, clinical implications, and future directions" Developmental Disabilities Research Reviews 14 268-275

Amador Campos J A, Forns Santacana M, Guàrdia Olmos J, Peró Cebollero M, 2006 "Estructura factorial y datos normativos del Perfil de atención y del Cuestionario TDAH para niños en edad escolar" Psicothema 18 696-703

Anderson R M, Rapport M D, Kofler M J, 2007 "Attention deficit/hyperactivity disorder and behavioral inhibition: A meta-analytic review of the stop-signal paradigm" Journal of Abnormal Child Psychology 35 745-758

APA, 2002 Diagnostic and Statistical Manual of Mental Disorders (text revision) (Washington, DC: American Psychiatric Association)

Aznar Casanova J A, 2010 "Test for assessment of the attention-deficit by perceptual rivalry", Registro de la Propiedad Intelectual No B-1055-10, Spain 
Barkley R A, 1997 ADHD and the Nature of Self-control (New York: Guilford Press)

Barkley R A, 2006 Attention-Deficit Hyperactivity Disorder: A Handbook for Diagnosis and Treatment 3rd edition (New York: Guilford Press)

Barkley R A, Murphy K R, 2006 Attention-Deficit Hyperactivity Disorders: A Clinical Workbook 3rd edition (New York: Guilford Press)

Blake R, 1989 “A neural theory of binocular rivalry” Psychological Review 96 145-167

Blake R, Logothetis N K, 2002 "Visual competition" Nature Reviews Neuroscience 3 13-21

Carlson C L, Mann M, 2002 "Sluggish cognitive tempo predicts a different pattern of impairment in the attention deficit hyperactivity disorder, primarily inattentive type" Journal of Clinical Child and Adolescent Psychology 31 123-129

Carter O, Cavanagh P, 2007 "Onset rivalry: Brief presentation isolates an early independent phase of perceptual competition" PLOS ONE 4 e343

Chong S C, Blake R, 2006 "Exogenous attention and endogenous attention influence initial dominance in binocular rivalry" Vision Research 46 1794-1803

Chong S C, Tadin D, Blake R, 2005 "Endogenous attention prolongs dominance durations in binocular rivalry" Journal of Vision 5 1004-1012

Conners C K, 1997 Conners'Rating Scales—Revised (Toronto, ON: Multi-Health Systems)

Diamond A, 2005 "Attention-deficit disorder (attention deficit/hyperactivity disorder without hyperactivity): A neurobiologically and behaviorally distinct disorder from attention-deficit/hyperactivity disorder (with hyperactivity)" Development and Psychopathology 17 807-825

Hancock S, Andrews T J, 2007 "The role of voluntary and involuntary attention in selecting perceptual dominance during binocular rivalry" Perception 36 288-298

Helmholtz H von, 1962 Treatise on Physiological Optics translated from 3rd German edition by J P C Southall (New York: Dover)

Kalisvaart J P, Rampersad S M, Goossens J, 2011 "Binocular onset rivalry at the time of saccades and stimulus jumps" PLoS ONE 6(6), e20017

Lamme V A, Roelfsema P R, 2000 "The distinct modes of vision offered by feedforward and recurrent processing" Trends in Neuroscience 23 571-579

Lee D K, Itti L, Koch C, Braun J, 1999 “Attention activates winner-take-all competition among visual filters" Nature Neuroscience 2 375-381

Lehky S R, 1988 “An astable multivibrator model of binocular rivalry” Perception 17 215-228

Leopold D A, Logothetis N K, 1996 "Activity changes in early visual cortex reflect monkeys' percepts during binocular rivalry" Nature 379 549-553

Levelt W J M, 1965 On Binocular Rivalry (Assen: Royal Van Gorcum)

Lijffijt M, Kenemans J L, Verbaten M N, Engeland H van, 2005 “A meta-analytic review of stopping performance in attention deficit/hyperactivity disorder: deficient inhibitory motor control? Journal of Abnormal Psychology 114 216-222

Logothetis N K, Leopold D A, Sheinberg D L, 1996 "What is rivaling during binocular rivalry?" Nature 300 621-624

Martinez-Conde S, Macknik S L, Troncoso X G, Hubel D H, 2009 “Microsaccades: a neurophysiological analysis" Trends in Neuroscience 32 463-475

Meng M, Tong F, 2004 "Can attention selectively bias bistable perception? Differences between binocular rivalry and ambiguous figures" Journal of Vision 4 539-551

Milich R, Balentine A C, Lynam D R, 2001 "ADHD combined type and ADHD predominantly inattentive type are distinct and unrelated disorders" Clinical Psychology: Science and Practice 8 463-488

Mitchell J F, Stoner G R, Reynolds J H, 2004 "Object-based attention determines dominance in binocular rivalry" Nature $\mathbf{4 2 9} 410-413$

Mueller T J, Blake R, 1989 "A fresh look at the temporal dynamics of binocular rivalry" Biological Cybernetics $61223-232$

Nigg J T, 2001 “Is ADHD an inhibitory disorder?” Psychological Bulletin 127 571-598

Ooi T L, He Z J, 1999 "Binocular rivalry and visual awareness: the role of attention" Perception 28 $551-574$

Oosterlaan J, Logan G D, Sergeant J A, 1998 "Response inhibition in AD/HD, CD, comorbid $\mathrm{AD} / \mathrm{HD}+\mathrm{CD}$, anxious and control children: A meta-analysis of studies with the stop task" Journal of Child Psychology and Psychiatry 39 411-425 
Pettigrew J D, 2001 "Searching for the switch: Neural bases for perceptual rivalry alternations" Brain and Mind 2 85-118

Rommelse N N J, Van der Stigchel S, Sergeant J A, 2008 "A review on eye movement studies in childhood and adolescent psychiatry" Brain and Cognition 68 391-414

Sergeant J A, Geurts H, Oosterlaan J, 2002 "How specific is a deficit of executive functioning for attention-deficit/hyperactivity disorder?" Behavioural Brain Research 130 3-28

Sheinberg D L, Logothetis N K, 1997 "The role of temporal cortical areas in perceptual organization" Proceedings of the National Academy of Sciences of the USA 94 3408-3413

Tong F, 2001 "Competing theories of binocular rivalry: A possible resolution" Brain and Mind 2 55-83 Wechsler D, 2003/2005 Wechsler Intelligence Scale for Children 4th edition (San Antonio, TX: Harcourt Assessment) [Escala de Inteligencia de Wechsler para Niños (Madrid: TEA)]

Wheatstone C, 1838 "Contributions to the physiology of vision - part the first. On some remarkable and hitherto unobserved phenomena of binocular vision" Philosophical Transactions of the Royal Society of London 128 371-394

Wolfe J M, 1983 "Afterimages, binocular rivalry, and the temporal properties of dominance and suppression" Perception 12 439-445 